\title{
Role of Apomorphine in the Treatment of Parkinson's Disease
}

\author{
Allison Boyle • William Ondo
}

Published online: 13 February 2015

(c) Springer International Publishing Switzerland 2015

\begin{abstract}
Current research shows that apomorphine is an effective treatment for symptoms of Parkinson's Disease (PD). The highly lipophilic structure allows apomorphine to cross cell membranes rapidly, leading to the rapid onset of action for on/off symptoms of PD. The use of apomorphine was limited in the past due to peripheral side effects, but with the advent of better delivery systems and medications to control side effects, apomorphine is better tolerated and more widely in use. The major delivery systems are continuous subcutaneous infusions and intermittent subcutaneous injections, but other delivery routes are under investigation. The purpose of this article is to discuss the current use of apomorphine, the current delivery systems and to discuss future research.
\end{abstract}

\section{Key Points}

Apomorphine is an effective symptomatic treatment for PD.

The highly lipophilic nature allows for rapid transit to the CNS.

Rapid onset of action allows for fast abortion of PD 'off' time symptoms.

Most common delivery methods are continuous or intermittent subcutaneous infusion/injection.

\footnotetext{
A. Boyle $\cdot$ W. Ondo $(\bowtie)$

University of Texas Health Science Center at Houston, 6410 Fannin Street, Ste 1010, Houston, TX 77030, USA

e-mail: William.Ondo@uth.tmc.edu
}

\section{A. Boyle}

e-mail: Allison.Boyle@uth.tmc.edu

\section{History}

Apomorphine was first synthesized in the mid-1800s after the discovery that morphine, once dehydrated with hydrochloric acid, created a new molecule, which was later named apomorphine [1, 2]. It was used initially in veterinary medicine to treat behavior deficits including stereotypies [1]. Later, apomorphine was used in human patients suffering from psychiatric conditions, alcohol and narcotic dependencies, as a sedative, as an emetic, for dystonia, and for chorea [1]. Apomorphine is one of the oldest medications that continue to be used clinically in medicine today. The first documented use for Parkinson Disease (PD) was in 1884. Use of apomorphine as an anti-Parkinson's agent, however, was limited by its short half-life, multiple side effects, the necessity for high doses if given orally, and nephrotoxicity [1]. It wasn't until the 1950s when it was discovered that the structure of apomorphine was similar to dopamine, and presumably acted at the dopamine receptor, that the drug was then reconsidered as a possible treatment option for PD. Clinical trials conducted with apomorphine as a therapy for PD at that time, concluded that due to its short duration of action and high level of "first-pass" hepatic metabolism, oral apomorphine was a poor treatment. These conclusions, coupled with the introduction of L-Dopa, relegated apomorphine to a minor therapeutic option for several more decades [2].

By the 1980s, limitations of L-Dopa had become apparent, especially fluctuations (dyskinesia, shorter duration of clinical effect with time) and inconsistent oral bioavailability. Researchers began a new line of apomorphine research, emphasizing injectable preparations, which proved to have more rapid onset and more consistent response than oral L-Dopa. Currently, continuous sub-cutaneous infusions, and intermittent subcutaneous injections 
are widely used, with other novel preparations in development [1, 3, 4].

\section{Chemistry}

Apomorphine [(6aR)-6-methyl-5, 6, 6a, 7-tetrahydro-4Hdibenzo [de,g]quinoline-10, 11-diol] shares a structural motif similar to dopamine, which is thought to confer its dopamine agonist (DA) properties [3, 4]. Chemically it is a derivative of morphine acidification and is designated: $6 \mathrm{a} \beta-$ Aporphine-10,11-diol hydrochloride hemihydrate with a molecular formula of $\mathrm{C} 17 \mathrm{H} 17 \mathrm{NO} 2$ [5]. Apomorphine is composed of 2 parts, the dopamine like moiety or catechol radical that binds to the dopamine receptor and the 2-amino-tetralein structure that determines the dopaminergic agonist property [4] (Fig. 1a, b). Its most distinct feature, compared to other DA, is its very lipophilic structure, resulting in its ability to cross membranes, including the blood brain barrier, passively. This results in very high and rapid concentrations of apomorphine in the brain, where it preferentially accumulates. Brain concentrations of apomorphine can be up to eight times higher than those in plasma [6]. The compound is acidic with a $\mathrm{pH}$ 3-4, which limits its practical absorption through mucosal tissue [7]. Current research of the chemical nature of apomorphine is being conducted using Raman Microscopy techniques and are analyzing apomorphine's chemistry to assist in developing novel approaches of detection of apomorphine, as well as better stabilizing approaches. Analyzing apomorphine with Raman Microscopy assists in detecting the apomorphine's plasma levels within a patient quickly and is a minimally invasive approach. The use of this technique will help researchers gain insight into the metabolism of apomorphine, in an effort to better determine the effectiveness of novel delivery routes [8].

Apomorphine is a non-selective dopamine receptor agonist, due to its binding affinities for all $5 \mathrm{DA}$ receptors, $D_{1}$-like receptors: $D_{1}$ and $D_{5}$, and $D_{2}$-like receptors: $D_{2 S}$, $\mathrm{D}_{2 \mathrm{~L}}, \mathrm{D}_{3}$, and $\mathrm{D}_{4}$. Compared to other oral DA's, it has greater D1 affinity, more closely resembling the profile of dopamine. Apomorphine also has binding affinities for adrenoreceptors and histamine receptors where it acts as an antagonist [2]. Apomorphine does not have any affinity for opioid receptors [9].

Apomorphine has also been shown to be a potent antioxidant and free radical scavenger, which may have potential neuroprotective effects. Apomorphine has been found in some studies to increase the activity of proteasomes and insulin-degrading enzymes in the brain that promote the degradation of intracellular Amyloid Beta (AB) peptides. $\mathrm{AB}$ peptide assembly in the brain forms amyloid plaques, which when accumulated have a<smiles>CN1CCc2cccc3c2[C@H]1Cc1ccc(O)c(O)c1-3</smiles><smiles>NCCc1ccc(O)c(O)c1</smiles>

Fig. 1 a Apomorphine structural formula. By Jü (Own work) [Public domain], via Wikimedia Commons (http://commons.wikimedia.org/ wiki/File:Apomorphine_Structural_Formulae.png\#file). b Dopamine structural formula. By Cacycle (Own work) [GFDL (http://www.gnu. org/copyleft/fdl.html), CC-BY-SA-3.0 (http://creativecommons.org/ licenses/by-sa/3.0/) or Public domain], via Wikimedia Commons (http:// commons.wikimedia.org/wiki/File:Dopamine_chemical_structure.png)

neurotoxic effect by promoting a chronic inflammatory state and increased free radial production $\left(\mathrm{H}_{2} \mathrm{O}_{2}, \mathrm{O}_{2}\right)$, apoptosis, and cell death. The potential action of apomorphine on formation of amyloid plaques has generated interest in exploring its therapeutic use for Alzheimer's disease [6, 10-12].

\section{Pharmocokinetics}

The oral availability of apomorphine is very poor, around $4 \%$ [13]. The parent compound is active whereas metabolites are not thought to have meaningful action upon dopamine receptors. Apomorphine has a rapid onset of action and brief duration of effect. The drug absorption, volume of distribution, plasma clearance and half-lives $(T 1 / 2)$ are similar for subcutaneous injection, subcutaneous infusion and IV infusion. Pharmacodynamic effects of apomorphine have been found to last up to $30 \mathrm{~min}$ after the plasma concentration has fallen to below the clinical efficiency threshold [14]. Serum metabolism is rapid $(T 1 / 2$ of 30-60 min, mean 43) with a high clearance $(3-5 \mathrm{~L} / \mathrm{kg} / \mathrm{h})$. Elimination is a two-compartment model with the main metabolic route auto-oxidation. Approximately $10 \%$ is transformed in the liver by glucuronidation and sulfation and only approximately $5 \%$ is excreted unchanged in the urine $[6,9,15]$. Of potential clinical interest, apomorphine is partially metabolized by catecholamine-O-methyl transferase (COMT), which also partially metabolizes LDopa, and can be inhibited with approved medications (entacapone and tolcapone). Tolcapone (COMT inhibitor) has been found to increase L-Dopa's duration of effect by inhibiting the enzymatic methylation of L-Dopa. One small 
pharmacokinetic/clinical study did show that tolcapone increased the serum area under the curve and duration of clinical improvement with an oral preparation of apomorphine [16].

\section{Early Clinical Data in PD}

The early work of use of apomorphine in PD patients was conducted mainly by Schwab et al. [17] in the 1950s. While these early trials showed apomorphine's efficacy for treatment of Parkinson symptoms, patients reported marked side effects and required high oral doses in order to see an effect. The drug languished until 1970, when fast acting, injectable subcutaneous preparations were developed [4, 18]. Patients with PD had a similar response in motor symptoms, including tremor, with apomorphine as compared to L-Dopa [4, 18]. That same year, Braham et al. showed improvement in tremor within 5-10 min lasting $1-2 \mathrm{~h}$ following subcutaneous injections of $0.5-2 \mathrm{mg}$ apomorphine in 11 of 15 Parkinson patients with L-Dopa resistant tremor. Side effects in the early trials consisted of syncope and vomiting, and were frequent [4, 19].

Additional apomorphine trials were performed in the late 1980s, both for diagnostic and therapeutic purposes. At subcutaneous doses ranging from 1 to $10 \mathrm{mg}$, authors reported a positive response in majority of idiopathic PD but no response in Parkinson plus syndromes [4, 20-23]. These trials were facilitated by the use of domperidone. This potent $\mathrm{D}_{2}$ antagonist does not cross the blood brain barrier, so can reduce peripheral side effects of apomorphine, especially nausea, without mitigating the desired CNS effect. It was usually given (10-20 mg) from 2 to $48 \mathrm{~h}$ prior to treatment with apomorphine, and is still used in most countries when initiating apomorphine [24, 25]. Trials have also been conducted using the anti-emetic trimethobenzamide, which works directly on the chemoreceptor trigger zone in the periphery. This drug is largely an anti-histamine with low affinities for dopamine receptors thus producing antiemetic effects without antidopaminergic properties [26]. The majority of the therapeutic trials conducted in the late 1980s and 1990s consistently demonstrated a reduction in the number of daily 'off' periods [4, 25, 27-31]. These trials also showed the majority of patients had a reduction in other 'off' phenomenon such as early morning dystonia, dysphagia, anismus, urinary dysfunction and 'off' period pain [4, 27, 28, 31-33].

\section{Current and Future Delivery Methods: Tolerability}

The most common current preparations are subcutaneous injections to abort "off" periods in patients taking L-Dopa and continuous subcutaneous infusions, which are administered throughout the day. Both have been used for many years but continue to be refined [9, 34]. Sublingual and nasal preparations have not been successfully developed to this point but new preparations are being tested [35-37]. Specific preparations have unique side effects but the drug itself has several consistent problems. The most common issues are nausea and vomiting, and hypotension including syncope. These tend to occur most dramatically with dose initiation, and improve with continued use, but often still result in discontinuation. Patients are typically pretreated with an anti-emetic in an effort to decrease nausea. In the past, the majority of trials pretreatment was with domperidone, but more recent US trials used trimethobenzamide as pretreatment because domperidone is not available [38]. Patients often yawn just prior to clinical improvement. This is an interesting, though not usually problematic side effect [3, 9]. Other side effects typical for DA's are also seen: sedation, hallucinations, and impulse control disorders.

\subsection{Intermittent Sub-Cutaneous Injections}

Motor fluctuations with L-Dopa, especially wearing off, affect a large population of patients with PD and contribute markedly to morbidity. One observational registry called Implications of Motor/fluctuations in Parkinson's Disease Patients on Chronic Therapy (IMPACT) found that the most significant quality of life problems reported by patients were loss of mobility and decreased ability to perform daily activities [35].

Three pivotal trials led to the approval of apomorphine subcutaneous injections for the treatment of motor fluctuations in PD in the US APO202, APO301 and APO302 were all prospective, randomized, placebo-controlled clinical trials conducted between 2001 and 2007. The trials all assessed the use of subcutaneous apomorphine $(10 \mathrm{mg} /$ $\mathrm{mL})$ as an acute treatment for "off" episodes in patients with idiopathic PD, and employed the Unified Parkinson's Disease Rating Scale (UPDRS) motor score from pre-dose to post-dose to assess the effect of apomorphine. They consistently found significant improvement in UPDRS scores after a mean time of 20 min versus placebo, which were generally similar in degree to L-Dopa [35, 37, 39, 40].

Between 1999 and 2005, an open label clinical trial, APO401, was conducted in an effort to study the safety and adverse effect profile of continued use of intermittent subcutaneous apomorphine injections used to treat "off" episodes. It enrolled 546 subjects with Hoehn and Yahr stage II-V PD and motor fluctuations despite optimal management with oral medications from 61 US sites. Most adverse effects experienced were mild to moderate and consisted of vomiting, dyskinesia, dizziness, somnolence, 
hallucinations, yawning and injection site bruising. The study concluded that the long-term use of intermittent apomorphine treatment for "off" episodes was safe [9].

A recent clinical trial, Apokyn for Motor Improvement of Morning Akinesia Trial (AM IMPAKT), studied the use of subcutaneous apomorphine injections specifically for patients with delayed onset of action of oral L-Dopa taken upon awakening. Not unexpectedly, subcutaneous injections more rapidly resulted in an initial "on" state compared to their previous oral L-Dopa [39, 41].

Onset of action with subcutaneous administration is extremely rapid, providing relief, on average, between 7 and 14 min post injection. The rate of uptake can be influenced by multiple factors including injection location, temperature, depth of injection, body fat and differences in enzymatic pathways between patients. Some studies have shown a 5-10 time difference in time to onset and concentration due to these variables [43]. Variation of onset between patients is high, but this variability is lower within individuals, meaning that injection site location and administration route, intermittent injection vs. continuous infusion, is less variable when considered in one patient than when comparing multi-patient studies/data $[7,9,42]$.

\subsection{Continuous Subcutaneous Infusions}

The other major use of apomorphine is continuous subcutaneously infusions throughout the day via a pump. As PD progresses, motor symptoms tend to fluctuate and "off" periods can become more unpredictable. Continuous infusion of apomorphine becomes indicated when patients progress to have increased amount of "off" time, motor fluctuations and dyskinesia [34, 42, 43]. Multiple long term studies have shown that PD patients have a significant reduction in motor fluctuations, significantly less "off" time, and use less oral L-Dopa with continuous apomorphine infusions [7, 42, 43]. Continuous subcutaneous infusion of apomorphine involves a pump and reservoir similar to that used for insulin infusions. From this pump a small catheter is inserted into the patient for infusions (Fig. 2). Infusions are typically started at $1 \mathrm{mg} / \mathrm{h}$ and are then titrated until optimal benefit. Usually they are stopped at night and restarted, often with a bolus, in the morning. Additional bolusing to allow the patient/care-giver to administer a rescue dose for the intermittent "off" periods is also possible $[7,9,43]$.

Studies, mostly open label series, suggest that continuous apomorphine can achieve steady on with or without concurrent L-Dopa. Studies have found that apomorphine monotherapy can reset the peak-dose dyskinesia threshold in L-Dopa treated patients, thus further decreasing fluctuations $[43,44]$. There are limited data comparing continuous apomorphine infusions to continuous L-Dopa

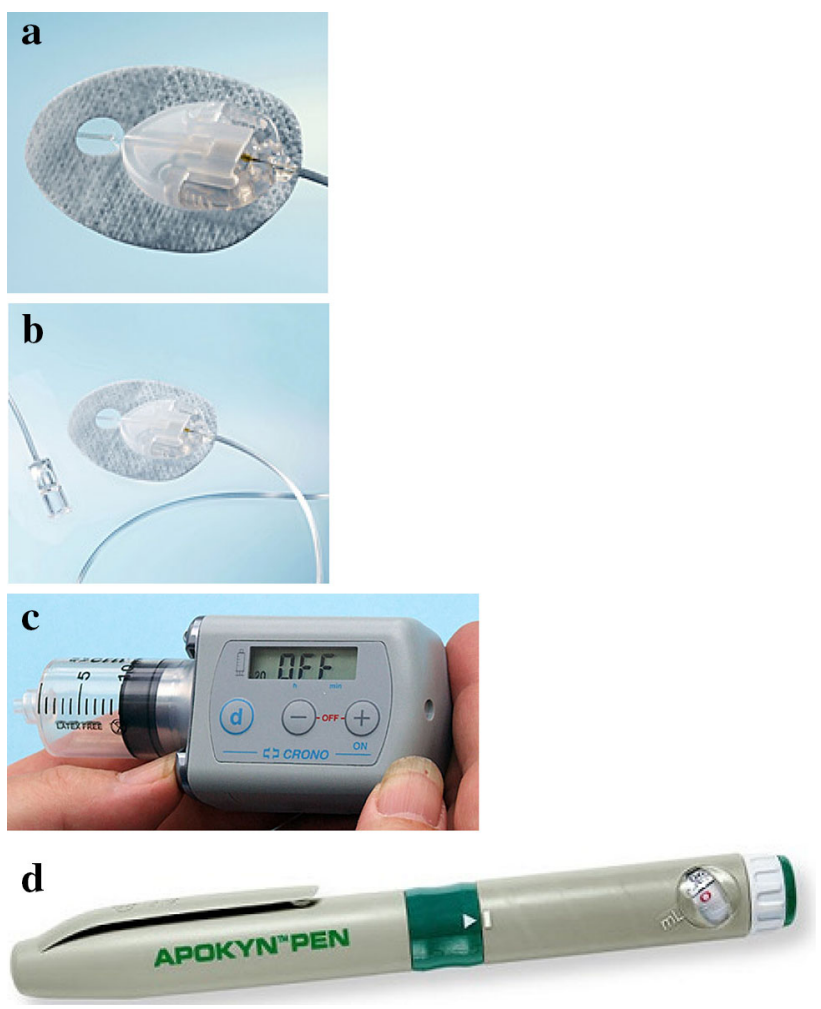

Fig. 2 a and b Images of the Neria soft needle, $\mathbf{c}$ image of the Cane Crono infusion pump (http://www.applied-medical.co.uk/soft.shtml), d Apokyn injector pen

jejunal pumps, but the few studies done seem to show less dyskinesia with apomorphine infusion [34].

Continuous infusions of apomorphine have been complicated by the development of skin nodules with prolonged therapy. Incidence of nodules is reduced with good skin hygiene and further reduced by the use of new needle technology utilizing a Teflon cannula inserted at $45^{\circ}$ angle compared to previous use of metal butterfly needle. The newest needles utilized are soft needles that use a Teflon cannula inserted at a $45^{\circ}$ angle that reduces tissue irritation [7, 34, 43] (Fig. 2).

\subsection{Intravenous Administration}

Intravenous routes of administration of apomorphine have also been investigated. Several studies summarized in Manson et al. [45], have looked at the use of IV formulations in patients that have been found to have refractory motor fluctuations and dermal complications from subcutaneous use. While studies show that patients do have a decrease in 'off' periods with IV administration, use is limited by severe adverse effects, including hypotension, syncope and skin infections. The more severe adverse effects that limited use are development of intravascular thrombosis thought to be due to the crystallization of 
apomorphine in the catheter. These are rarely used now [6, 46].

\subsection{Oral Administration}

Orally administered apomorphine is subject to nearly complete first pass hepatic metabolism [47]. The bioavailability of oral delivered apomorphine is therefore extremely low, and in order to achieve therapeutic levels, very high doses are required, which may increase the frequency of adverse events, such as nausea and nephrotoxicity in some cases [4, 18, 47]. No commercial oral preparation is currently used.

\subsection{Sublingual and Nasal Administration}

Due to the chemical nature of apomorphine and its highly oxidative property, finding a means to stabilize apomorphine for delivery is essential. The oxidation of the molecule is increased with an alkaline environment, light and oxygen. This has constrained routes of delivery, due to apomorphine's rapid degeneration in solutions intended for either oral or nasal administration. The highly acidic properties of apomorphine also contribute to stoma formation in oral/sublingual administrations, as well as severe nasal lining irritation. Research has been done investigating different systems that utilize stabilizing buffers integrated with the active solid apomorphine in an effort to reduce the acidity [39, 48].

Sublingual formulations have shown clinical efficacy in the past but were complicated by mucosal irritation [49]. Recently, two new promising routes for delivering apomorphine have emerged and are being clinically studied, a new sublingual formulation and a new powdered formulation for nasal insufflation. The sublingual administration is based on the Listerine breath strips, named APL130277, and will start trials in 2015 [39]. In this formulation, a thin film delivery system is utilized incorporating active solid apomorphine in a stabilizing buffer that allows for less acidity. The thin strip rapidly dissolves in saliva allowing for quick absorption with less mucosal irritation due to buffer solution. This novel sublingual delivery route is currently in stage II clinical trials and seems to overcome some issues previously observed with sublingual apomorphine while achieving rapid onset of action as observed in subcutaneous injections of apomorphine [39, 48]. There are many potential advantages to a sublingual administration including less need for manual dexterity as is needed with subcutaneous IV administration, no need for needle, no potential skin irritation and pain [39]. The trial will have several studies included in it including a pilot study to determine the effect of APL-130277 on relieving the "off" episodes in PD pts, the bridging study to compare the bioavailability of sublingual formulation to subcutaneous formulation, an efficacy study and a safety study [39].

A new insufflation system that utilizes the method of time-of-administration mixing to activate a less irritating, but also less stable, form of apomorphine is currently under clinical investigation [50]. Early results and observances have found this novel nasal delivery has similarly rapid onset of action, while minimizing adverse events, specifically nasal irritation. In addition to nasal delivery systems, new pulmonary delivery systems are also being investigated. Pulmonary inhalation would allow for larger surface area leading to rapid absorption [50, 51].

\section{Efficacy of Apomorphine for Non-Motor Features of PD}

Non-motor symptoms (NMS) of PD are now known to occur throughout all stages of the disease and negatively impact the quality of life of these patients. NMS include neuropsychiatric and gastrointestinal (GI) symptoms, sleep disturbances [including Restless Leg Syndrome (RLS)], urinary dysfunction, pain and impulse control disorders. While no formal studies have been conducted looking at the direct effect of apomorphine on NMS, there is enough evidence from the numerous trials focused on motor symptoms of PD to suggest a beneficial effect of continuous infusion of apomorphine on several NMS in patients with PD [43]. Focused studies need to be explored in the future.

\section{Other Uses (Erectile Dysfunction, RLS, and Neuroprotection)}

Other DA's robustly treat the symptoms of RLS. A few case reports by Tribl et al. [52] show efficacy of apomorphine in RLS. The short $T \frac{1}{2}$ relegates this to treating breakthrough symptoms in severe cases, unless continuous infusions are used.

Apomorphine has been long known to act as an erectogenic agent due to its action on dopamine receptors in the CNS. The dopamine receptors in the CNS that are involved in sexual function are mainly the $\mathrm{D}_{2}$ like receptors in the putamen, thalamus, medial preoptic area nucleus and the paraventricular nucleus, which then project to the spinal cord [6]. Efficacy results were mixed and complicated by typical side effects. The development of effective cGMPspecific phosphodiesterase-5 inhibitors such as sildenafil has diminished interest for this indication [4].

Studies have been done looking at the use of apomorphine in patients in a minimally conscious or persistent vegetative state with encouraging results. Studies have 
used both subcutaneous injections and infusions in patients and have seen improvement with both formulations [53, 54].

Apomorphine's potent antioxidant and free radial scavenger properties have created interest in possible neuroprotective effects in patients with neurodegenerative conditions such as PD as well as Alzheimer's disease. The cause of Alzheimer's disease is not well understood, but two main brain changes are seen: intracellular neurofibrillary tangles and extracellular senile plaques. Apomorphine has been seen in preliminary studies to promote degradation of beta amyloid plaques and lessen the burden of hyperphosphorylated tau. It is not known whether patients with $\mathrm{AD}$ will have a similar adverse effect profile as those with PD, but it postulated that the dose of apomorphine needed for neuroprotection may be less than that needed for PD. Further research is needed [6, 11, 12].

\section{Conclusion}

Current research shows that apomorphine is an effective treatment for symptoms of PD in either an intermittent subcutaneous injection or continuous subcutaneous infusion. The highly lipophilic structure and subcutaneous administration allows apomorphine to cross cell membranes rapidly leading to the rapid onset of action for on/off symptoms of PD. The use of apomorphine was limited in the past due to peripheral side effects, namely nausea and hypotension, but with the advent of better delivery systems and medications to control side effects, apomorphine is better tolerated and more widely in use, although still generally reserved for advanced patients with motor fluctuations and severe "off" times. The major delivery systems currently utilized are continuous subcutaneous infusions and intermittent subcutaneous injections. Future research is aimed at developing new delivery systems with less complications and the use of apomorphine in other conditions such as RLS and dementia.

Acknowledgments William Ondo has received grant support from USWorld Meds, HSG and Lundbeck Dystonia Coalition; consultant fees from ACADIA, Auspex, Lundbeck, USWorld Meds and UCB Pharma; and speaker fees from TEVA, Merz, Lundbeck, UCB Pharma and Avanir. Allison Boyle does not have any conflicts of interest to declare. No funding were received to prepare this review.

\section{References}

1. Hagell P, Odin P, Shing M. Apomorphine in Parkinson's disease. UNI-MED-Verlag, 2008.

2. Gessa GL, Corsini GU. Apomorphine and other dopaminomimetics: basic pharmacology. New York: Raven Press (ID); 1981.
3. Stacy M, Silver D. Apomorphine for the acute treatment of "off" episodes in Parkinson's disease. Parkinsonism Relat Disord. 2008;14(2):85-92.

4. Muguet D, Broussolle E, Chazot G. Apomorphine in patients with Parkinson's disease. Biomed Pharmacother Biomed Pharmacother. 1995;49(4):197-209.

5. Apomorphine. Wikipedia, The Free Encyclopedia May 6, 2014 [cited 2014 June 6]. Available from: http://en.wikipedia.org/w/ index.php?title $=$ Apomorphine\&oldid $=607359824$.

6. Ribaric S. The pharmacological properties and therapeutic use of apomorphine. Molecules. 2012;17(5):5289-309.

7. Pietz K, Hagell P, Odin P. Subcutaneous apomorphine in late stage Parkinson's disease: a long term follow up. J Neurol Neurosurg Psychiatry. 1998;65(5):709-16.

8. Lucotti A, Tommasini M, Casella M, Morganti A, Gramatica F, Zerbi G. TLC - surface enhanced Raman scattering of apomorphine in human plasma. Vib Spectrosc. 2012;62:286-91.

9. LeWitt PA, Ondo WG, Van Lunen B, Bottini PB. Open-label study assessment of safety and adverse effects of subcutaneous apomorphine injections in treating "off" episodes in advanced Parkinson disease. Clin Neuropharmacol. 2009;32(2):89-93.

10. Himeno E, Ohyagi Y, Ma L, Nakamura N, Miyoshi K, Sakae N, et al. Apomorphine treatment in Alzheimer mice promoting amyloid-beta degradation. Ann Neurol. 2011;69(2):248-56.

11. Gassen M, Gross A, Youdim MBH. Apomorphine enantiomers protect cultured pheochromocytoma (PC12) cells from oxidative stress induced by $\mathrm{H}_{2} \mathrm{O}_{2}$ and 6-hydroxydopamine. Mov Disord. 1998;13(4):661-7.

12. Gassen M, Glinka Y, Pinchasi B, et al. Apomorphine is a highly potent free radical scavenger in rat brain mitochondrial fraction. Eur J Clin Pharmacol. 1996;308:219-26.

13. Gancher ST, Nutt JG, Woodward WR. Absorption of apomorphine by various routes in parkinsonism. Mov Disord Off J Mov Disord Soc. 1991;6(3):212-6.

14. Holford NH, Sheiner LB. Understanding the dose-effect relationship: clinical application of pharmacokinetic-pharmacodynamic models. Clin Pharmacokinet. 1981;6(6):429-53.

15. Holford NG, Sheiner L. Understanding the dose-effect relationship. Clin Pharmacokinet. 1981;6(6):429-53.

16. Ondo WG, Hunter C, Vuong KD, Jankovic J. The pharmacokinetic and clinical effects of tolcapone on a single dose of sublingual apomorphine in Parkinson's disease. Parkinsonism Relat Disord. 2000;6(4):237-40.

17. Schwab RS, Amador LV, Lettvin JY. Apomorphine in Parkinson's disease. Trans Am Neurol Assoc. 1951;56:251-3.

18. Cotzias GC, Papavasiliou PS, Tolosa ES, Mendez JS, BellMidura M. Treatment of Parkinson's disease with aporphines. Possible role of growth hormone. $N$ Engl J Med. 1976;294(11):567-72.

19. Braham J, Sarova-Pinhas I, Goldhammer Y. Apomorphine in Parkinsonian tremor. Br Med J. 1970;3(5725):768.

20. Barker R, Duncan J, Lees A. Subcutaneous apomorphine as a diagnostic test for dopaminergic responsiveness in parkinsonian syndromes. Lancet. 1989;1(8639):675.

21. Oertel, Wolfgang $\mathrm{H}$, et al. Apomorphine test for dopaminergic responsiveness. Lancet. 1989;333(8649):1262-3.

22. Hughes AJ, Lees AJ, Stern GM. Apomorphine test to predict dopaminergic responsiveness in parkinsonian syndromes. Lancet. 1990;336(8706):32-4.

23. D'Costa DF, Abbott RJ, Pye IF, Millac PA. The apomorphine test in parkinsonian syndromes. J Neurol Neurosurg Psychiatry. 1991;54(10):870-2.

24. Factor SA. Literature review intermittent subcutaneous apomorphine therapy in Parkinson's disease. Neurology. 2004;62(6 suppl 4):S12-7. 
25. Steiger MJ, Quinn NP, Marsden CD. The clinical use of apomorphine in Parkinson's disease. J Neurol. 1992;239(7):389-93.

26. Bowron A. Practical considerations in the use of apomorphine injectable. Neurology. 2004;62(6 suppl 4):S32-6.

27. Stibe CM, Lees AJ, Kempster PA, Stern GM. Subcutaneous apomorphine in parkinsonian on-off oscillations. Lancet. 1988;1(8582):403-6.

28. Poewe W, Kleedorfer B, Gerstenbrand F, Oertel W. Subcutaneous apomorphine in Parkinson's disease. Lancet. 1988;1(8591):943.

29. Chaudhuri KR, Critchley P, Abbott RJ, Pye IF, Millac PA. Subcutaneous apomorphine for on-off oscillations in Parkinson's disease. Lancet. 1988;2(8622):1260.

30. Pollak P, Champay AS, Gaio JM, Hommel M, Benabid AL, Perret J. Subcutaneous administration of apomorphine in motor fluctuations in Parkinson's disease. Revue Neurol. 1990;146(2):116-22.

31. Frankel JP, Lees AJ, Kempster PA, Stern GM. Subcutaneous apomorphine in the treatment of Parkinson's disease. J Neurol Neurosurg Psychiatry. 1990;53(2):96-101.

32. Petit H, Vermersch P, Caparros-Lefebvre D. Subcutaneous apomorphine in major motor oscillations of parkinsonian patients. Presse Med. 1992;21(4):172.

33. Christmas TJ, Kempster PA, Chapple CR, Frankel JP, Lees AJ, Stern GM, et al. Role of subcutaneous apomorphine in parkinsonian voiding dysfunction. Lancet. 1988;2(8626-8627):1451-3.

34. Clarke CE, Worth P, Grosset D, Stewart D. Systematic review of apomorphine infusion, levodopa infusion and deep brain stimulation in advanced Parkinson's disease. Parkinsonism Relat Disord. 2009;15(10):728-41.

35. Stacy M, Spears J, Van Lunen B. Implications of Motor fluctuations in Parkinson's patients on Chronic Therapy (IMPACT) registry: comparison of patient and physician reported data. Mov Disord. 2006;21(Supplement 13):1.

36. Montastruc JL, Rascol O, Senard JM, Gualano V, Bagheri H, Houin G, et al. Sublingual apomorphine in Parkinson's disease: a clinical and pharmacokinetic study. Clin Neuropharmacol. 1991;14(5):432-7.

37. Dewey RB Jr, Hutton JT, LeWitt PA, Factor SA. A randomized, double-blind, placebo-controlled trial of subcutaneously injected apomorphine for parkinsonian off-state events. Arch Neurol. 2001;58(9):1385-92.

38. Hauser RA, Isaacson S, Clinch T. Randomized, placebo-controlled trial of trimethobenzamide to control nausea and vomiting during initiation and continued treatment with subcutaneous apomorphine injection. Parkinsonism Relat Disord. 2014;20(11):1171-6.

39. Anthony Giovinazzo M, Dir C, CEO CT, Agro IA, Bryson N, Tankosic IT. Apomorphine for off periods in Parkinson's disease: clinical use and potential of a developmental sublingual formulation, APL130277. In: Conference M, editor. 2013.
40. Pfeiffer RF, Gutmann L, Hull KL Jr, Bottini PB, Sherry JH, Investigators APOS. Continued efficacy and safety of subcutaneous apomorphine in patients with advanced Parkinson's disease. Parkinsonism Relat Disord. 2007;13(2):93-100.

41. Isaacson S, Ondo W, Pagan F. Apomorphine improves morning akinesia in Parkinson's disease: interim analysis of the AMIMPAKT trial. European Journal of Neurology; 2014: WileyBlackwell 111 River St, Hoboken 07030-5774, NJ USA; 2014. p. 68.

42. Garcia Ruiz PJ, Sesar Ignacio A, Ares Pensado B, Castro Garcia A, Alonso Frech F, Alvarez Lopez M, et al. Efficacy of long-term continuous subcutaneous apomorphine infusion in advanced Parkinson's disease with motor fluctuations: a multicenter study. Mov Disord Off J Mov Disord Soc. 2008;23(8):1130-6.

43. Todorova A, Chaudhuri KR. Subcutaneous apomorphine and non-motor symptoms in Parkinson's disease. Parkinsonism Relat Disord. 2013;19(12):1073-8.

44. Manson AJ, Turner K, Lees AJ. Apomorphine monotherapy in the treatment of refractory motor complications of Parkinson's disease: long-term follow-up study of 64 patients. Mov Disord Off J Mov Disord Soc. 2002;17(6):1235-41.

45. Manson A, Hanagasi H, Turner K, Patsalos P, Carey P, Ratnaraj $\mathrm{N}$, et al. Intravenous apomorphine therapy in Parkinson's disease clinical and pharmacokinetic observations. Brain $\mathrm{J}$ Neurol. 2001;124(2):331-40.

46. Gancher ST, Woodward WR, Boucher B, Nutt JG. Peripheral pharmacokinetics of apomorphine in humans. Ann Neurol. 1989;26(2):232-8.

47. LeWitt PA. Subcutaneously administered apomorphine: pharmacokinetics and metabolism. Neurology. 2004;62(6 Suppl 4):S8-11.

48. Garrido JMPJ, Delerue-Matos C, Borges F, Macedo TRA, Oliveira-Brett AM. New insights into the oxidation pathways of apomorphine. J Chem Soc Perkin Trans 2. 2002;10:1713-7.

49. Ondo W, Hunter C, Almaguer M, Jankovic J. A novel sublingual apomorphine treatment for patients with fluctuating Parkinson's disease. Mov Disord Off J Mov Disord Soc. 1999;14(4):664-8.

50. Morgan FG, Main MJ. Compositions and uses. Google Patents; 2013.

51. Bartus RT, Emerich DF. Pulmonary delivery in treating disorders of the central nervous system. Google Patents; 2003.

52. Tribl GG, Sycha T, Kotzailias N, Zeitlhofer J, Auff E. Apomorphine in idiopathic restless legs syndrome: an exploratory study. J Neurol Neurosurg Psychiatry. 2005;76(2):181-5.

53. Fridman EA, Krimchansky BZ, Bonetto M, Galperin T, Gamzu ER, Leiguarda RC, et al. Continuous subcutaneous apomorphine for severe disorders of consciousness after traumatic brain injury. Brain Inj. 2010;24(4):636-41.

54. Pistoia F, Mura E, Govoni S, Fini M, Sarà M. Awakenings and awareness recovery in disorders of consciousness. CNS Drugs. 2010;24(8):625-38. 